\title{
Lymphoma B-cell responsiveness to CpG-DNA depends on the tumor microenvironment
}

\author{
Rym Ben Abdelwahed ${ }^{1,2,3,8,9}$, Jérémie Cosette ${ }^{1,2,3,4}$, Sabrina Donnou ${ }^{1,2,3,5,6,7}$, Lucile Crozet ${ }^{1,2,3}$, Hanane Ouakrim ${ }^{1,2,3}$, \\ Wolf Herman Fridman ${ }^{1,2,3}$, Catherine Sautès-Fridman ${ }^{1,2,3}$, Aouni Mahjoub $b^{8,9}$ and Sylvain Fisson ${ }^{1,2,3,5,6,7 *}$
}

\begin{abstract}
Background: Toll-like receptor (TLR) agonists have important properties that can be exploited for immunotherapy against tumors. Locally injected immunostimulatory oligodeoxynucleotides containing CpG motifs (CpG-ODNs), which are TLR9 agonists, have shown promise in cancer models. Several studies have demonstrated that these motifs have immunologic effects similar to those of bacterial DNA and can stimulate monocytes, macrophages, dendritic, and B cells, which then produce several proinflammatory cytokines. However, these CpG-ODNs appear to produce opposite effects on tumor B cells.

Methods: In this study, we investigated the direct effects of a murine class B CpG (1826) ODNs on lymphoma B cells in vitro and in vivo, using mouse models of non-Hodgkin B lymphomas developing in immunoprivileged sites, specifically the brain and the eye, and in subcutaneous sites.

Results: In vitro, CpG-ODNs produced antiproliferative and proapoptotic effects on lymphoma B cells. In vivo, it had an antitumor effect when injected into tumors in murine models of subcutaneous lymphoma (SCL) and primary cerebral lymphoma (PCL). However, its intravitreal administration into a primary intraocular lymphoma (PIOL) mouse model did not produce an antitumor effect. In vitro experiments using supernatant from mouse PIOL samples demonstrated that the PIOL molecular microenvironment inhibits the antiproliferative effect of CpG-ODNs on lymphoma B-cells.
\end{abstract}

Conclusions: Responsiveness to $\mathrm{CpG}$ stimulation differs in subcutaneous, cerebral, and ocular tumors, according to the tumoral and molecular microenvironment, and this should be considered for further therapeutic approaches.

Keywords: TLR, CpG-DNA, Non-Hodgkin B-cell lymphoma, Subcutaneous lymphoma, Primary cerebral lymphoma, Primary intraocular lymphoma, Tumor microenvironment

\section{Background}

Toll-like receptors (TLRs) are pattern recognition receptors that trigger innate and adaptive immune responses. Triggering TLRs activates a set of common proinflammatory genes and leads to the expression of antimicrobial effector cells and to production of inflammatory cytokines [1]. Agonists for TLRs have been identified and are being developed as new drugs and vaccine adjuvants to treat cancer, allergies, and infectious diseases [2]. In particular, oligodeoxynucleotides containing CpG motifs (CpG-ODN),

\footnotetext{
* Correspondence: sylvain.fisson@univ-evry.fr

${ }^{1}$ Institut National de la Santé et de la Recherche Médicale (INSERM), UMRS872, Centre de Recherche des Cordeliers, Paris F-75006, France ${ }^{2}$ Université Pierre et Marie Curie-Paris 6, UMRS 872, Paris F-75006, France Full list of author information is available at the end of the article
}

which are TLR9 agonists, have shown promise against several types of tumors, including renal cell carcinoma, glioblastoma, melanoma, cutaneous T-cell lymphoma, and non-Hodgkin lymphoma [3]. Unmethylated CpG-DNA motifs have immunologic effects similar to those of bacterial DNA and can stimulate monocytes, macrophages, and dendritic and B cells; these then produce several Th1-type cytokines [4].

At least 3 structurally distinct classes of synthetic CpGODNs have been described, all capable of stimulating cells that express TLR9 [5,6]. CpG-B (also known as class-B CpG or K-type $\mathrm{CpG}$ ) ODNs encode multiple CpG motifs on a phosphorothioate backbone and trigger the differentiation of antigen-presenting cells and the proliferation

\section{() Biomed Central}

(c) 2013 Ben Abdelwahed et al.; licensee BioMed Central Ltd. This is an Open Access article distributed under the terms of the Creative Commons Attribution License (http://creativecommons.org/licenses/by/2.0), which permits unrestricted use, distribution, and reproduction in any medium, provided the original work is properly cited. 
and activation of B cells [3]. Although the CpG-B motif is an established immunostimulatory agent, its direct effect on normal and tumor B cells seems to differ: CpG-ODNs stimulate proliferation of healthy $B$ cells, activate their production of polyreactive immunoglobulins, and protect them from apoptosis [6-8]. On the other hand, these ODNs predominantly activate malignant $\mathrm{B}$ cells and then increase the rate of cell death, thus reducing survival of malignant B cells over time [9-11].

Different types of non-Hodgkin B-cell lymphomas differ in their responsiveness to CpG-DNA, and only limited information is available [9] about the sensitivity of malignant $\mathrm{B}$ cells to this DNA motif according to their in vivo microenvironment, particularly in immune sanctuaries such as the brain and eyes. Unlike systemic lymphoma, primary cerebral lymphoma (PCL) and primary intraocular lymphoma (PIOL) are subsets of primary central nervous system lymphoma (PCNSL), and they affect immunologically privileged organs. Both usually appear as a diffuse large B-cell non-Hodgkin lymphoma in which malignant lymphoid cell types not normally present in the brain or eye are detected [12]. The internal tissues of the brain and eye are usually protected from the inflammatory processes mediated by the immune system.

In this study, we compare the effect of CpG-ODNs on cerebral and ocular diffuse large B-cell lymphoma and on subcutaneous lymphomas (SCL). We show that A20.IIA murine B-cell lymphoma expressed high levels of endogenous TLR9 protein that produced an antiproliferative effect when stimulated in vitro by CpG-ODNs. A proapoptotic effect accompanied this reduced proliferation. In vivo local administration had a similar antitumor effect on subcutaneous and cerebral lymphomas. However, local administration of CpG-ODNs in a PIOL mouse model did not produce an antitumor effect. In vitro experiments with supernatant from ocular lymphoma samples demonstrated that the molecular microenvironment of PIOL counteracts the direct antiproliferative effect of CpG-ODNs on lymphoma B-cells. These findings show that cerebral and ocular tumor cells differ in their responsiveness to $\mathrm{CpG}$ stimulation according to the tumor environment. The microenvironment of the eye must be further characterized to identify the negative regulators.

\section{Methods \\ Reagents}

Nuclease-stable phosphorothioate-modified CpG 1826 (CpG) with 5_-TCCATGACGTTCCTGACGTT (the nucleotides in bold represent the immunostimulatory $\mathrm{CpG}$ sequences), fluorescein isothiocyanate (FITC)-conjugated CpG 1826 ODNs, and control 1826 ODN with 5_-TCC ATGAGCTTCCTGAGCTT were purchased from InvivoGen (Cayla, France).

\section{Cells}

A20.IIA is an Fc $\gamma R$-negative clone originating from the A20-2 J B-cell lymphoma line [13]. For in vivo experiments, A20.IIA cells were transfected by an electroporation system with the green fluorescent protein (GFP) gene. These cells, hereafter referred to as A20.IIA or A20. IIA-GFP cells as appropriate, were maintained at $37^{\circ} \mathrm{C}, 5 \%$ $\mathrm{CO}_{2}$ in complete Roswell Park Memorial Institute (RPMI) 1640 Medium Glutamax plus (RPMI; Gibco-Invitrogen, France) supplemented with $10 \%$ fetal calf serum (FCS; PAA Laboratories, Germany), $100 \mu \mathrm{g} / \mathrm{mL}$ penicillin and $100 \mu \mathrm{g} / \mathrm{mL}$ streptomycin (both from Eurobio, France), $10 \mathrm{mM}$ sodium pyruvate (Gibco-Invitrogen), and $50 \mu \mathrm{M}$ 2-mercaptoethanol (Gibco-Invitrogen). The A20.IIA-GFP cell culture was also supplemented with $0.5 \mathrm{mg} / \mathrm{mL}$ neomycin (G418; Gibco-Invitrogen). To obtain the A20. IIA-luc2 cell line, A20.IIA cells were transfected with pGL4.50[luc2/CMV/hygro] (Promega), in the AMAXA Nucleofector II device (Lonza, Switzerland) and were cultured in $0.75 \mathrm{mg} / \mathrm{mL}$ hygromycin B (Gibco-Invitrogen) medium.

\section{Proliferation assay}

A20.IIA cells at a concentration of $10^{5} \mathrm{cells} / \mathrm{mL}$ were incubated with serial dilutions of CpG 1826 or control 1826 ODNs at concentrations ranging from 0.0003 to $60 \mu \mathrm{g} / \mathrm{mL}$ or with complete RPMI medium alone. After 3 days, $\left[{ }^{3} \mathrm{H}\right]$ thymidine (GE Healthcare) was added for the last $4 \mathrm{~h}$. Cells were harvested onto fiber filters and $\left[{ }^{3} \mathrm{H}\right]$ thymidine incorporation was measured in a scintillation counter (Microbeta, Perkin Elmer).

\section{Apoptosis assay}

A20.IIA cells $\left(10^{4}\right)$ were cultured in complete RPMI medium in 96-well plates in the presence or absence of $3 \mu \mathrm{g} / \mathrm{mL}$ or $30 \mu \mathrm{g} / \mathrm{mL}$ of CpG or control ODNs. Staining with Annexin V/allophycocyanin (APC) and propidium iodide (PI) (BD Biosciences, France) was performed $72 \mathrm{~h}$ later and then analyzed by flow cytometry. Apoptotic cells were defined as those positive for Annexin V and PI.

\section{Mice}

Female BALB/c mice $\left(\mathrm{H}-2^{\mathrm{d}}\right)$ were obtained from Charles River Laboratories (L'Arbresle, France) and used between 6 and 8 weeks of age. They were provided with sterile food and water ad libitum and kept on a 12-hour lightdark cycle. All procedures involving mice conformed with European Union guidelines, French regulations for animal experimentation (Ministry of Agriculture Act No. 2001-464, May 2001), and the guidelines of the Institut National de la Santé et de la Recherche Médicale Committee on Animal Research, and were approved by the relevant local committees (Charles Darwin Ethics Committee 
for Animal Experiments, Paris, France; Permit Number: p3/2009/004).

\section{Tumor implantation}

Mice were first anesthetized by intraperitoneal injection of a mixture containing $120 \mathrm{mg} / \mathrm{kg}$ of ketamine (Virbac, France) and $6 \mathrm{mg} / \mathrm{kg}$ of xylazine (Rompun 2\%; Bayer Healthcare). To obtain a subcutaneous lymphoma (SCL) murine model, BALB/c mice were inoculated subcutaneously with $5 \times 10^{6}$ A20.IIA-GFP tumor cells in a final volume of $50 \mu \mathrm{L}$ of RPMI, at 2 different sites: the right and left abdomen. For the intracerebral tumor implantation, anesthetized mice were immobilized on a stereotaxic frame (David Kopf Instruments, Tujunga, CA, USA). Tumor cells $\left(5 \times 10^{4}\right.$ in a final volume of $2 \mu \mathrm{L}$ RPMI) were injected into the specific cerebral location (right striatum), located $2 \mathrm{~mm}$ to the right of the medial suture and $0.4 \mathrm{~mm}$ in front of the bregma, through a Hamilton syringe attached to a penetrating depth controller. The penetrating depth of the syringe was $2.5 \mathrm{~mm}$ from the surface of the brain. Each injection delivered the solution slowly, and the syringe was held in place for an additional minute to reduce backfilling of tumor cells. For the intravitreal tumor implantation, we used a 32gauge needle attached to a syringe to inject $10^{4}$ cells in a final volume of $2 \mu \mathrm{L}$ of RPMI into the vitreous under a dissecting microscope. Lacrinorm 2\% (Bauch\&Lomb) drops were instilled after intravitreal injection. For each tumor model, control mice received either $1 \times$ phosphatebuffered saline (pH7.4; PBS) or control 1826 ODNs instead of CpG 1826 ODNs.

\section{Treatment injections}

Tumor growth in the SCL model was monitored by caliper measurements 3 times a week. Treatment began when the longest tumor diameter reached 0.5 to $0.7 \mathrm{~cm}$. The mice then received daily intratumor injections of CpG-ODNs for 5 days $(100 \mu \mathrm{g}$ per injection in a final volume of $50 \mu \mathrm{L}$ RPMI) in the right tumor only; the left tumor served as an untreated control tumor. Mice were killed one week after the last treatment injection. Lymphomas established in the brain and eye were treated 7 days after tumor inoculation, by a single local injection of $60 \mu \mathrm{g}$ (brain) or $20 \mu \mathrm{g}$ (eye) CpG-ODNs in $2 \mu \mathrm{L}$ of RPMI (treatment groups) or $2 \mu \mathrm{L}$ of PBS (control groups). Tumor burden was analyzed in the sacrificed mice one week after treatment administration.

\section{Isolation of brain, ocular and subcutaneous lymphomas}

The tumor-injected brains and eyes and the subcutaneous tumors were harvested one week after treatment injection, minced with surgical scissors, incubated for 30 minutes in RPMI containing $0.1 \mathrm{mg} / \mathrm{mL}$ DNAse I (Roche Diagnostics, Meylan, France) and 1.67 Wünch
$\mathrm{U} / \mathrm{mL}$ Liberase (Roche), and filtered through a $70-\mu \mathrm{m}$ membrane (BD Falcon). Mononuclear cells were separated from myelin with a Percoll cell density gradient.

\section{In vivo tumor growth assay}

The A20.IIA $\left(1 \times 10^{4}\right)$ cells expressing luciferase (luc2 gene) were injected via subcutaneous, intracerebral or intravitreal routes into immunocompetent 7-week-old $\mathrm{BALB} / \mathrm{c}$ mice. CpG or control ODNs were administered in situ for each lymphoma model according to the same experimental design and at the time points and doses described above. The tumor burden was thereafter monitored by bioluminescence imaging. Mice were injected intraperitoneally with $150 \mathrm{mg} / \mathrm{kg}$ of D-luciferin potassium salt (Interchim) and underwent imaging within the next 10 minutes with the IVIS LUMINA II (Caliper LS) imaging system. The exposure time was set to optimize the signal and obtain the best signal-to-noise ratio. The bioluminescence signal is expressed in photons per second.

\section{Supernatant harvesting}

Mice were implanted with tumor cells in the brain (PCL), eye (PIOL) or flank (SCL) or injected with PBS in the eye (PIE). Either 14 days later (brain and eye) or when tumor diameter reached 0.5 to $0.7 \mathrm{~cm}(\mathrm{SCL})$, the relevant cells were isolated and cultured for $36 \mathrm{~h}$ in complete RPMI medium in 96 -well $\left(10^{4}\right.$ cells per well) plates. Supernatant was then harvested from each well.

\section{Flow cytometry}

To analyze TLR9 expression on A20.IIA cells, these cells underwent intracellular staining with the Fixation/ Permeabilization solution kit (BD Biosciences) and an anti-TLR9/PE mAb (BD Biosciences).

Tumor burden was analyzed according to the following protocol: Fc receptors were saturated for $20 \mathrm{~min}$ with $10 \mu \mathrm{g} / \mathrm{mL}$ of anti-CD16/CD32 mAb (clone 2.4.G2), and then the cells were incubated for 20 min with either rat IgG2a anti-CD19/APC mAb, or the corresponding isotypic $\mathrm{mAb}$ control (all from BD Biosciences). The living cells were defined with side scatter (SSC) and forward scatter (FSC) after autofluorescent cells were excluded. Cell phenotypes were analyzed with the LSRII cytometer and Diva software (BD Biosciences).

\section{Statistical analysis}

Comparisons used Student's t-test, performed with GraphPad Prism (GraphPad Software, La Jolla, CA, USA). Statistical significance was defined by $p$ values less than 0.05 . 


\section{Results}

CpG-ODNs inhibit cell proliferation and induce apoptosis of malignant A20.IIA B cells in vitro

TLR9 is an intracellular receptor that recognizes CpGDNA. Cell stimulation by $\mathrm{CpG}$ motifs requires that they bind to TLR9. We therefore began by confirming with flow cytometry that A20.IIA B lymphoma cells express TLR9 (Figure 1A).

We next evaluated the direct effect of CpG-ODNs on the proliferation of A20.IIA lymphoma in vitro. Based on our study of its proliferation kinetics (data not shown), tumor cells were incubated for $72 \mathrm{~h}$ with CpG 1826 ODNs at concentrations ranging from 0.0003 to $30 \mu \mathrm{g} / \mathrm{mL}$. Cell proliferation was measured with the $\left[{ }^{3} \mathrm{H}\right]$ thymidine incorporation assay. The CpG-ODNs inhibited A20.IIA [ $\left.{ }^{3} \mathrm{H}\right]$ thymidine incorporation in a dose-dependent manner, whereas control ODNs had no effect on cell proliferation (Figure 1B). The maximum inhibitory effect was obtained from 0.3 to $30 \mu \mathrm{g} / \mathrm{mL}$ of CpG-ODNs.

Based on these results, we analyzed the induction of apoptosis of A20.IIA cells by CpG- ODNs and found that they induced apoptosis of about 30 to $35 \%$ of the cells (that is, 30-35\% stained positive for Annexin V and PI), compared with 5\% for RMPI Medium and 5-10\% with the control ODNs (Figure 1C).
Lymphoma cell responsiveness to CpG sequences differs according to their tissue microenvironment

After showing that the $\mathrm{CpG}$ motif has a direct antiproliferative and proapoptotic effect on A20.IIA lymphoma cells, we sought to explore its effects in vivo when injected intratumorally, by comparing the 3 types of murine models of lymphoma: SCL, PCL and PIOL. A20.IIA-GFP cells were implanted on the left and right flank of the mice for the SCL model. Tumor size was measured by a caliper 3 times a week. When the tumors reached $5-7 \mathrm{~mm}$ in diameter, the left site was treated by local injections of CpG-ODNs, while the right one was used as an untreated control tumor. As described by Houot \& Levy in 2009 [14] mice did or did not receive daily intratumoral injections of $100 \mu \mathrm{g} / 50 \mu \mathrm{L}$ CpG-ODNs for 5 days. Tumor size was then measured daily until sacrifice, one week after the last treatment injection. The tumor burden of mice treated with $\mathrm{CpG}$ and control ODNs was compared with a bioluminescence imaging system that assessed total photon influx. The CpGODNs inhibited tumor growth very soon after treatment in this SCL model. On day 7 after treatment, the untreated tumor was more than 100 times brighter than the CpG-treated one, and on day 20, 120 times brighter (Figure 2A). Flow cytometric analysis of $\mathrm{CD}_{19}{ }^{+} \mathrm{GFP}^{+}$

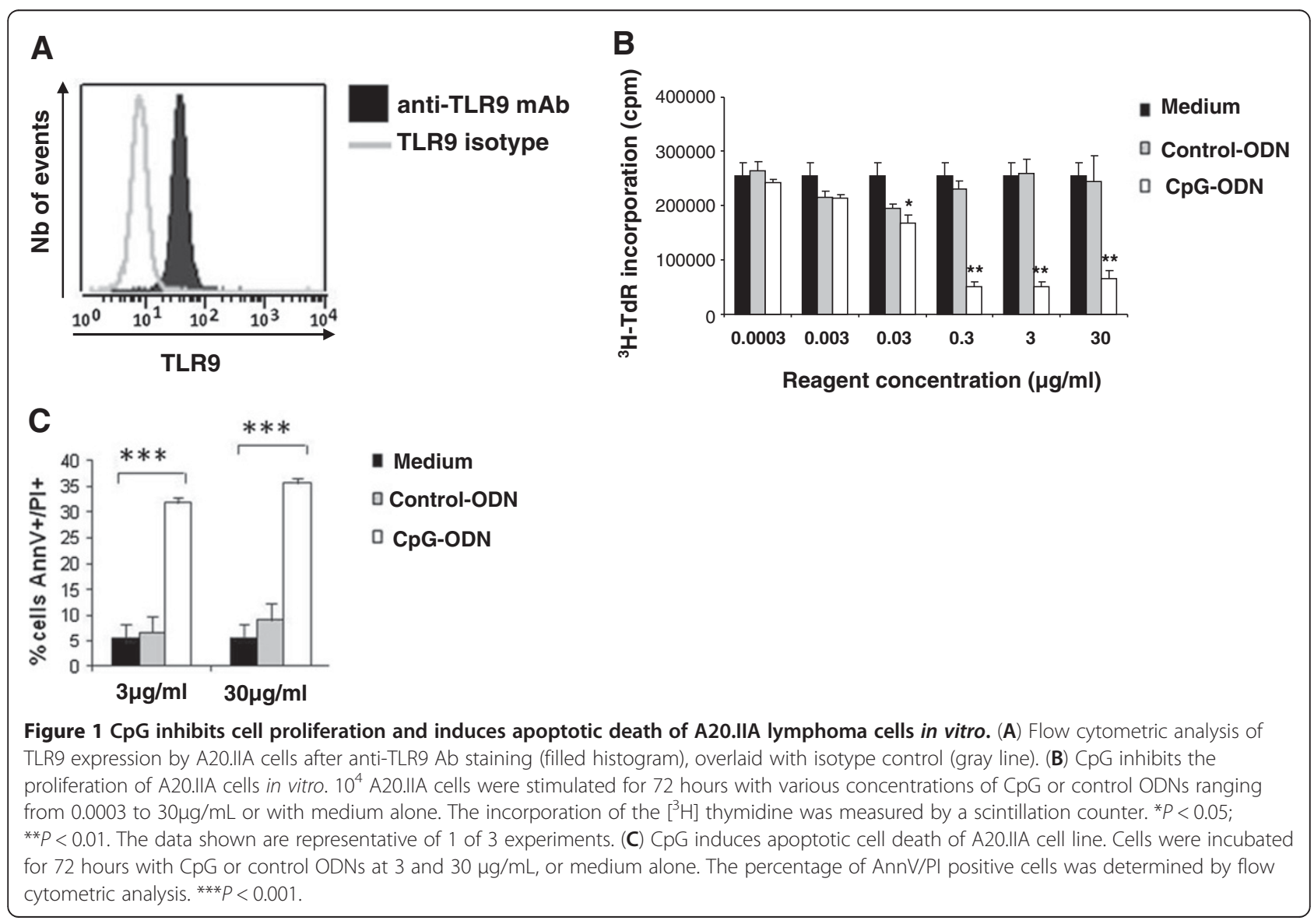




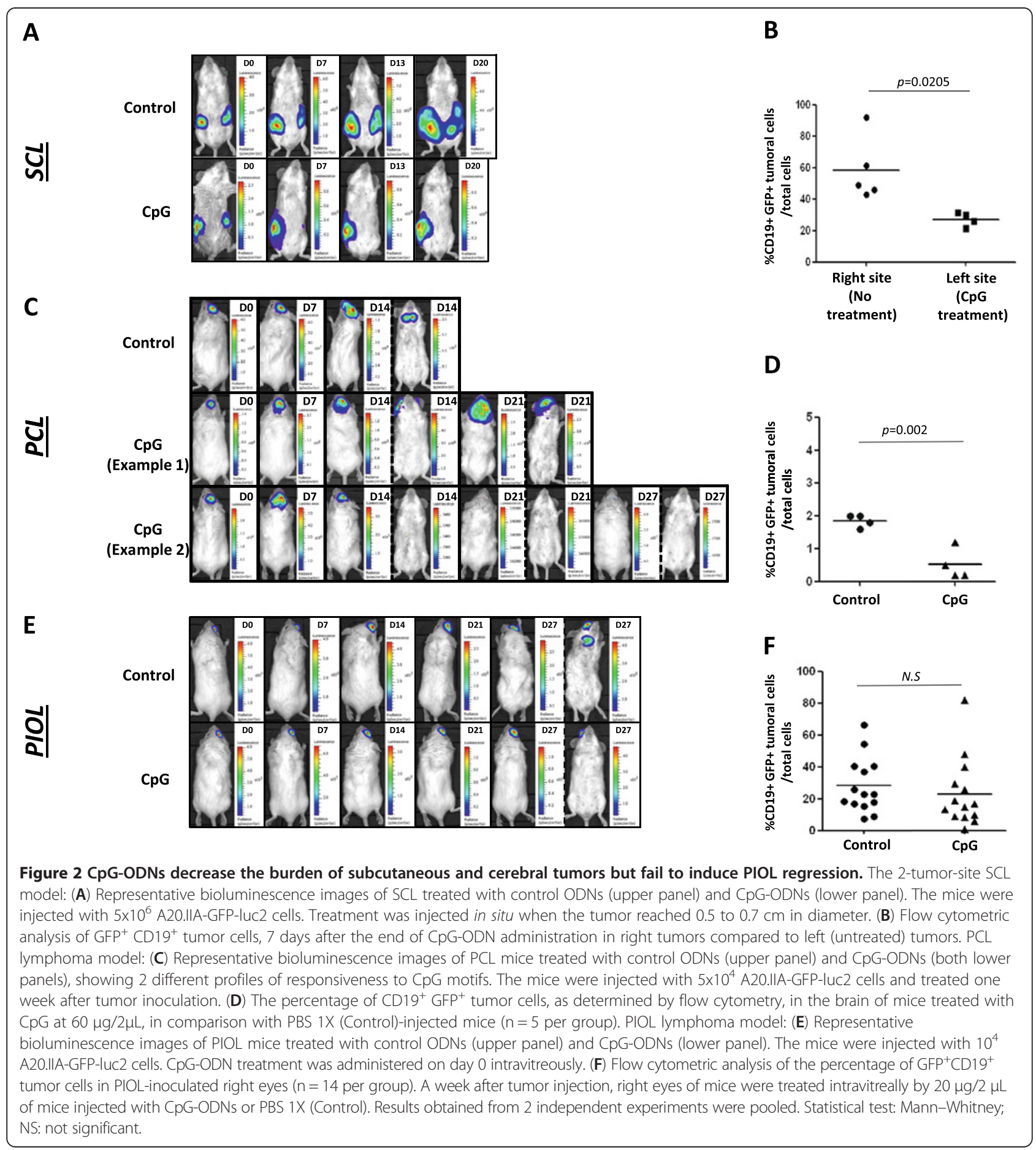

cells confirmed that tumor cells decreased significantly more in the treated than the untreated tumors (Figure 2B).

We next addressed the question of whether CpG motifs have the same antitumor effect in cerebral lymphomas. Imaging analysis showed two different profiles. Some mice did not respond to in situ CpG-ODN treatment, and the lymphoma developed in the brain and even developed in lymph nodes at day 21; this timing was nonetheless later than in the control group (Figure 2C - Example 1). Some mice did respond to the treatment; the tumor grew from day 0 to day 7 after treatment, and then decreased until it was undetectable (Figure 2C - Example 2). We also examined the percentage of $\mathrm{CD} 19^{+} \mathrm{GFP}^{+}$cells in the group treated by CpG-ODNs, compared it with the control group and observed a significant decrease in the proportion of tumor cells (Figure 2D). 
Next we investigated the antitumor effect of CpGODNs on PIOL mice that had a tumor implanted in the right eye only and were then treated with CpG-ODNs $(20 \mu \mathrm{g} / 2 \mu \mathrm{L})$ or control ODNs $(20 \mu \mathrm{g} / \mu \mathrm{L})$. As shown in Figure 2E, CpG-ODNs seem to have had no detectable effects on the primary eye tumor. Nevertheless, they appeared to prevent lymph node invasion at day 27 (Figure 2E). Flow cytometric analysis showed no significant difference in tumor growth between CpG ODNtreated and control (PBS 1X) treated eyes (Figure 2F).

These results suggest that the behavior of tumors in the eye is different from that of systemic lymphomas, but also from that of cerebral lymphoma, and thus, that tumor cells responsiveness to CpG-DNA depend on the tissue microenvironment.

\section{Soluble molecules from the PIOL microenvironment} counteract the antiproliferative effect of CpG-ODNs on malignant B-cells in a dose-dependent-manner

As described above, in vivo experiments showed that the responsiveness of lymphoma $\mathrm{B}$ cells to $\mathrm{CpG-ODN}$ administration was tissue-dependent. To confirm that the blockade of CpG-ODN antitumor effects was due to the PIOL molecular microenvironment, we tested whether supernatant from PIOL could counteract the inhibitory effect of CpG-ODNs on the proliferation of A20.IIA cells in vitro. A $\left[{ }^{3} \mathrm{H}\right]$ thymidine incorporation assay was performed as described above, with the addition of supernatant obtained from PBS-injected eyes (PIE) (as control), or from the mouse model SCL, PCL, and PIOL. As shown in Figure 3, the addition of PIE (Figure 3A) and SCL (Figure 3B) supernatants did not modify the ability of CpG-ODN treatment to inhibit tumor growth. PCL supernatant (Figure $3 \mathrm{C}$ ) increased proliferation, but $\mathrm{CpG}$ ODNs were still active at doses of 30 and $60 \mu \mathrm{g} / \mathrm{mL}$. In contrast, CpG-ODNs were unable to inhibit tumor cell proliferation after incubation with PIOL supernatant (Figure 3D) and to induce apoptosis (data not shown). Moreover, their antiproliferative effect was recovered upon dilution of the PIOL supernatant (Figure 4). These data confirm our in vivo results and show that a soluble factor, released in eye tumors but not in normal eyes, was able to counteract the antiproliferative effect of $\mathrm{CpG}$ motifs.

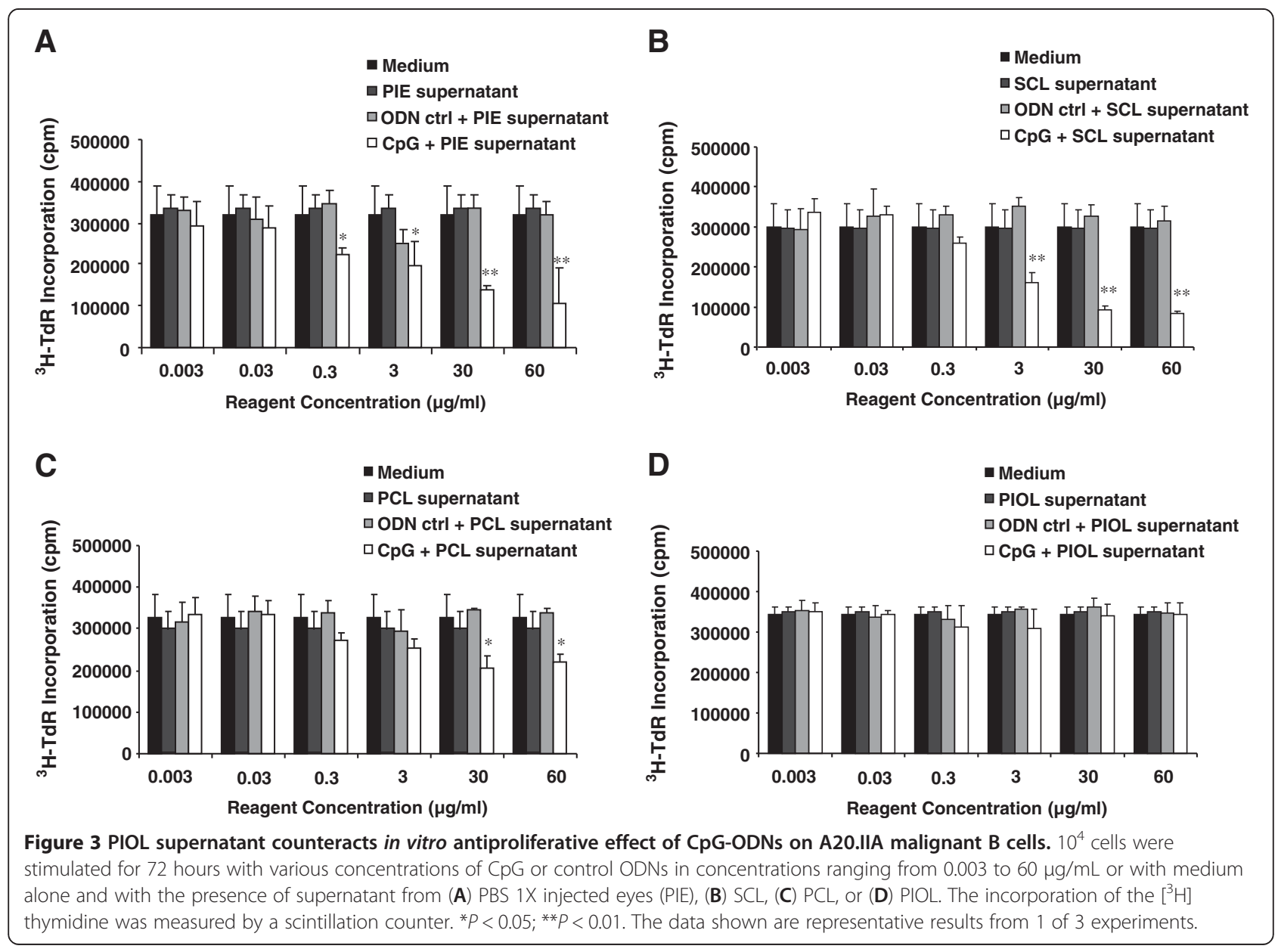




\section{Control supernatant}
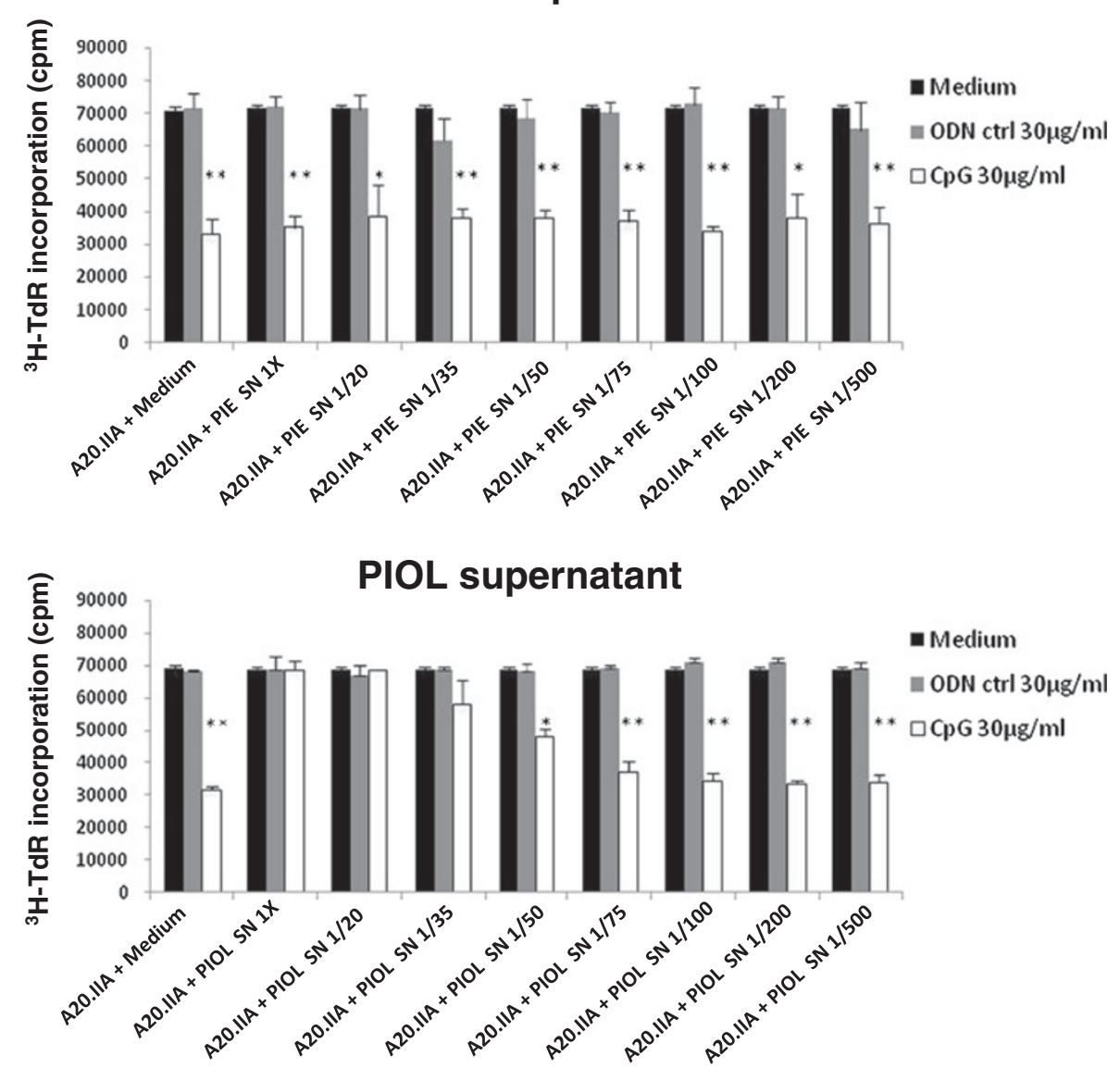

Figure 4 Soluble molecule present in PIOL but not in normal ocular microenvironment is able to abrogate in vitro effect of CpG-ODNs in a dose-dependent manner. $10^{4}$ cells were stimulated for 72 hours with $\mathrm{CpG}$ or control ODNs at $30 \mathrm{\mu g} / \mathrm{mL}$ and in the presence of several diluted doses of control supernatant (PIE) or PIOL supernatant $(1 \mathrm{X}, 1 / 20,1 / 35,1 / 50,1 / 75,1 / 100,1 / 200,1 / 500)$. The incorporation of the [ $\left.{ }^{3} \mathrm{H}\right]$ thymidine was measured by a scintillation counter. ${ }^{*} P<0.05 ;{ }^{*} P<0.01$.

The PIOL microenvironment did not modify either TLR9 expression or the internalization of CpG-ODNs by tumor cells

To investigate the possibility that the loss of the CpGODNs antitumor action was associated with modulation of TLR9 expression, we used flow cytometry to compare TLR9 expression on A20.IIA cells after incubation with supernatant from medium alone, PIOL or PIE. No differences were found between these conditions (Figure 5A).

Next we examined whether the PIOL molecular microenvironment inhibited internalization of CpG ODNs by tumor cells. FITC-labelled CpG 1826 ODNs were added for 24 hours at a concentration of $3 \mu \mathrm{g} / \mathrm{mL}$ to A20.IIA lymphoma cells in the presence of PIOL or PIE supernatant. Flow cytometric analysis indicated that FITC expression by tumor cells with PIOL supernatant was similar to that incubated with PIE supernatant (Figure 5B).These findings show that the addition of PIOL supernatant does not modify CpG internalization by lymphoma B-cells, even in vivo in our three model (data not shown).

\section{Discussion}

The ultimate goal of cancer immunotherapy is to eradicate tumors through vaccine strategies. Recent studies have demonstrated that synthetic CpG-ODNs induce regression of highly immunogenic tumors by engaging both the innate and the adaptive immune systems. CpG-ODNs are currently being tested in clinical trials for the treatment of non-Hodgkin B-cell lymphoma, which expresses TLR9 [15]. However, only limited information is currently available about the sensitivity to CpG-ODNs of primary malignant B-cells of different non-Hodgkin lymphoma entities. Understanding their direct effect on malignant B-cells is important as we consider how this potent class of agents might be used in the immunotherapy of lymphoma.

Here, we found that A20.IIA malignant murine cells, related to diffuse large B cells, express TLR9 and 

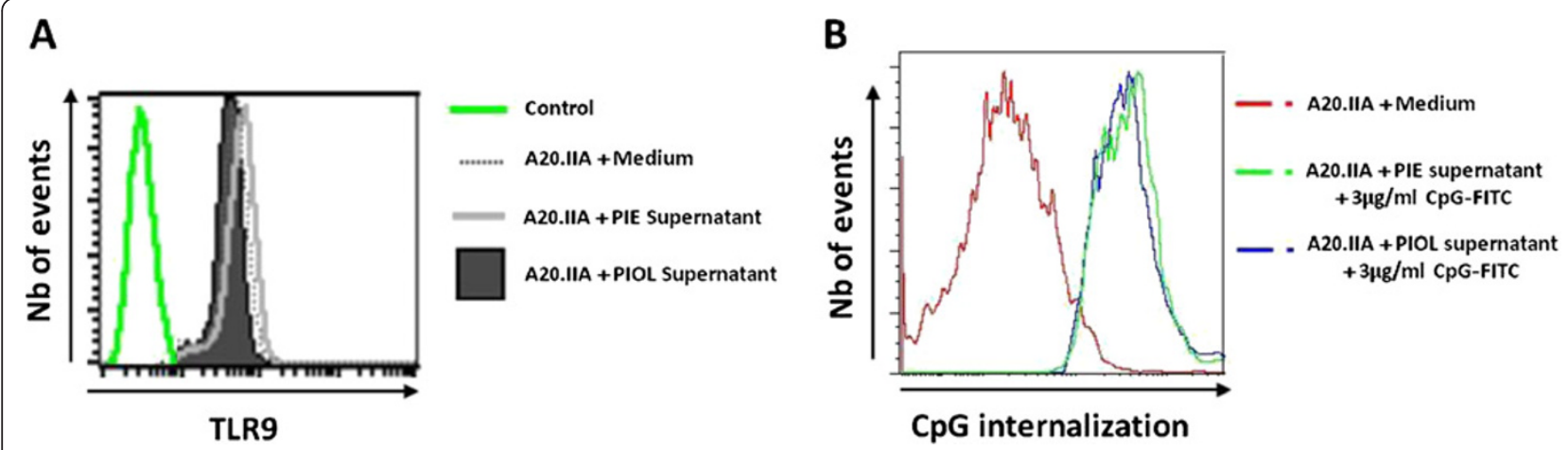

Figure 5 The PIOL microenvironment did not modify TLR9 expression or internalization of CpG-ODNs by tumor cells. (A) $10^{4}$ A20.IIA cells were incubated with PIOL or PIE supernatant. 3 days later, cytometric analysis was performed of TLR9 expression by cells incubated with PIOL supernatant, overlaid with isotype control and compared to TLR9 expression by cells incubated with PIE supernatant or medium alone. (B) $10^{4}$ A20.IIA cells were incubated for 24 hours with medium alone or with PIOL or PIE supernatant and in the presence or absence of FITClabeled CpG-ODNs at $3 \mu \mathrm{g} / \mathrm{mL}$. FITC expression by A20.IIA tumor cells was analyzed by flow cytometry.

are sensitive to CpG-B ODN stimulation in vitro. As reported previously, CpG-ODNs induce a dose-dependent antiproliferative effect [16] and increase apoptotic cell death [17]. This apoptosis has been described as caspasedependent and is accompanied by up-regulation of CD95/ Fas and its ligand [9]. Another group demonstrated that TLR9 signaling by CpG-B ODNs leads to NF-kB-dependent production of autocrine IL-10, which then activates JAK/ STAT pathway-dependent tyrosine phosphorylation of STAT1 proteins and thereby engenders an apoptotic pathway in human chronic lymphocytic leukemia B-cells [10]. Comparing primary B-cell lymphomas from patient samples, other authors have showed that cell responsiveness to CpG-ODNs varies, with different degrees of activation and apoptosis induction [9]. Several studies have reported that CpG-ODNs induce activation of normal B-cells and block apoptosis [7]. Although the molecular mechanisms of these effects remain unclear, it has been suggested that reactive oxygen species (ROS) and NFkB activation may play a role [18].

An important question is whether the in vitro responses to CpG motifs that have been observed could produce an in vivo antitumor effect on DLBCL lymphoma mouse models. We used 3 mouse models to begin to answer this question: a primary systemic lymphoma model (subcutaneous lymphoma) and 2 primary central nervous system lymphoma subtypes (cerebral and ocular lymphoma mouse models). The brain and eyes, considered to be immune sanctuaries, are relatively isolated from the systemic immune system by anatomic and physiologic barriers that maintain a local immune tolerance to protect neuronal cells from inflammation [19]. The use of these different models allowed us to compare the responsiveness to $\mathrm{CpG-ODNs}$ of the same tumor cells located in different immune microenvironments.
Thus, we demonstrated that local administration of CpG-ODNs into subcutaneous lymphoma decreased the tumor burden. This effect is probably attributable to immune cell activation of NK cells and DCs, which activates innate and adaptive immunity. In addition, the CpG-ODNs inhibited proliferation and induced apoptosis of TLR9-positive tumor cell lines in vitro. Interestingly, intratumor administration of CpG-ODNs induced tumor regression of cerebral but not intraocular lymphoma. The similarity in origin and classification of PCL and PIOL, which are related subsets sharing the particularity of developing in different immune-privileged sites, makes these results especially striking.

In addition, PIOL supernatant selectively abrogated the inhibitory effects of CpG-ODNs in vitro, in contrast to supernatant from nonmalignant eyes (PBS-injected eye) or SCL. PCL supernatant, on the other hand, had an intermediate inhibitory effect on the in vitro antiproliferative action of CpG-ODNs. Together, these data suggest that soluble factors are produced in the PIOL microenvironment, to a lesser degree in the PCL microenvironment, and not at all in subcutaneous microenvironment. These factors can inhibit the effect of this TLR9 agonist on lymphoma B-cells. This inhibition was not due to downregulation of TLR9 expression or to a blockade of CpG internalization by tumor cells. Further investigation is needed to characterize TLR9-mediated signaling and molecular mechanisms that might differ in the PIOL microenvironment.

\section{Conclusions}

In conclusion, we showed here that, in addition to their immune-enhancing effects, CpG-ODNs inhibit lymphoma B cell proliferation and induce apoptotic cell death in vitro. They also reduced tumor growth in systemic 
and cerebral lymphomas in vivo. These findings support the value of developing TLR9-targeted therapy with CpG-B ODNs as a therapeutic agent for primary nonHodgkin B-cell lymphoma. Further investigation should seek to identify and characterize the soluble factors from the PIOL microenvironment that inhibit the effects of CpG-ODNs and enable us to understand the potential immunosuppressive effect on host immune response that the ocular lymphoma microenvironment appears to produce.

\section{Abbreviations \\ APC: Allophycocyanin; CpG: Cytosine phosphodiester guanine; FITC: Fluorescein isothiocyanate; GFP: Green fluorescent protein; $\mathrm{H}$ - TdR: Tritiated thymidine; mAb: monoclonal antibody; ODN: Oligodeoxynucleotide; PBS: Phosphate buffered saline; PCL: Primary cerebral lymphoma; PCNSL: Primary central nervous system lymphoma; PE: Phycoerythrin; PI: Propidium iodide; PIE: PBS-injected eye; PIOL: Primary intraocular lymphoma; RPMI: Roswell Park Memorial Institute; SCL: Subcutaneous lymphoma; TLR: Toll-like receptor.}

\section{Competing interests}

The authors declare they have no financial conflicts of interest.

\section{Authors' contributions}

Contribution: RBA, JC, and SD performed the experiments and wrote the paper. LC and HO provided technical assistance; WHF, CSF, MA, and SF contributed to the writing and to the critical reading of the paper; SF conceived and planned the study. All authors read and approved the final manuscript.

\section{Acknowledgments}

Flow cytometry acquisition took place at the cellular imaging and cytometry platform (CICC, Centre de Recherche des Cordeliers, Paris F-75006, France). We are grateful to Jo Ann Cahn for her careful reading of the manuscript.

\section{Grant support}

This work was supported by the Institut National du Cancer (Grants RC013C06N631-2005 and C06N748-2006), the Association pour la Recherche contre le Cancer (ARC), the Institut National de la Santé et de la Recherche Médicale (INSERM), the University Pierre and Marie Curie (UPMC, Convergence project), the University Paris-Descartes, the pole de compétitivité lle de France (ImmuCan project), the Tunisian Direction Générale de la Recherche Scientifique, and the French-Tunisian DGRS-INSERM and CMCU (Egide-PHC Utique) projects. RBA received grants from the DGRS-INSERM and the CMCU. S.D. received a grant from the Institut National du Cancer (INCa).

\footnotetext{
Author details

${ }^{1}$ Institut National de la Santé et de la Recherche Médicale (INSERM), UMRS872, Centre de Recherche des Cordeliers, Paris F-75006, France. ${ }^{2}$ Université Pierre et Marie Curie-Paris 6, UMRS 872, Paris F-75006, France. ${ }^{3}$ Université Paris Descartes, UMRS 872, Paris F-75006, France. ${ }^{4}$ Laboratoire MSC, Université Paris 7/CNRS, Paris, France. ${ }^{5}$ Genethon, Evry, France. ${ }^{6}$ INSERM, U951, Evry, France. ${ }^{7}$ University of Evry Val d'Essonne, UMRS 951, 1 bis rue de I'Internationale, Evry F-91002, France. ${ }^{8}$ Faculté de Pharmacie de Monastir, LR99 ES27, Monastir 5000, Tunisie. ' Université de Monastir, Monastir 5000, Tunisie.
}

Received: 22 December 2012 Accepted: 30 March 2013 Published: 5 April 2013

\section{References}

1. Chang ZL: Important aspects of Toll-like receptors, ligands and their signaling pathways. Inflamm Res 2010, 59(10):791-808.

2. Dunne A, Marshall NA, Mills KH: TLR based therapeutics. Curr Opin Pharmacol 2011, 11(4):404-411.

3. Krieg AM: Toll-like receptor 9 (TLR9) agonists in the treatment of cancer. Oncogene 2008, 27(2):161-167.
4. Weiner GJ, Liu HM, Wooldridge JE, Dahle CE, Krieg AM: Immunostimulatory oligodeoxynucleotides containing the CPG motif are effective as immune adjuvants in tumor antigen immunization. Proc Natl Acad Sci USA 1997, 94(20):10833-10837.

5. Verthelyi D, Ishii KJ, Gursel M, Takeshita F, Klinman DM: Human peripheral blood cells differentially recognize and respond to two distinct CPG motifs. J Immunol 2001, 166(4):2372-2377.

6. Hartmann G, Krieg AM: Mechanism and function of a newly identified CpG DNA motif in human primary B cells. J Immunol 2000, 164(2):944-953.

7. Krieg AM, Yi AK, Matson S, Waldschmidt TJ, Bishop GA, Teasdale R, Koretzky GA, Klinman DM: CpG motifs in bacterial DNA trigger direct B-cell activation. Nature 1995, 374(6522):546-549.

8. Kuo CC, Liang CM, Lai CY, Liang SM: Involvement of heat shock protein (Hsp) 90 beta but not Hsp90 alpha in antiapoptotic effect of CpG-B oligodeoxynucleotide. J Immunol 2007, 178(10):6100-6108.

9. Jahrsdorfer B, Muhlenhoff L, Blackwell SE, Wagner M, Poeck H, Hartmann E, Jox R, Giese T, Emmerich B, Endres S, et al: B-cell lymphomas differ in their responsiveness to CpG oligodeoxynucleotides. Clin Cancer Res 2005, 11(4):1490-1499.

10. Liang X, Moseman EA, Farrar MA, Bachanova V, Weisdorf DJ, Blazar BR, Chen W: Toll-like receptor 9 signaling by CpG-B oligodeoxynucleotides induces an apoptotic pathway in human chronic lymphocytic leukemia B cells. Blood 2010, 115(24):5041-5052.

11. Jahrsdorfer B, Jox R, Muhlenhoff $L$, Tschoep K, Krug A, Rothenfusser $S$, Meinhardt G, Emmerich B, Endres S, Hartmann G: Modulation of malignant $B$ cell activation and apoptosis by bcl-2 antisense ODN and immunostimulatory CpG ODN. J Leukoc Biol 2002, 72(1):83-92.

12. Rubenstein J, Ferreri AJ, Pittaluga S: Primary lymphoma of the central nervous system: epidemiology, pathology and current approaches to diagnosis, prognosis and treatment. Leuk Lymphoma 2008, 49(Suppl 1):43-51.

13. Donnou S, Galand C, Touitou V, Sautes-Fridman C, Fabry Z, Fisson S: Murine models of B-cell lymphomas: promising tools for designing cancer therapies. Adv Hematol 2012, 2012:701-704.

14. Houot R, Levy R: T-cell modulation combined with intratumoral CpG cures lymphoma in a mouse model without the need for chemotherapy. Blood 2009, 113(15):3546-3552.

15. Weiner GJ: The immunobiology and clinical potential of immunostimulatory CpG oligodeoxynucleotides. J Leukoc Biol 2000, 68(4):455-463.

16. Li J, Song W, Czerwinski DK, Varghese B, Uematsu S, Akira S, Krieg AM, Levy $\mathrm{R}$ : Lymphoma immunotherapy with $\mathrm{CpG}$ oligodeoxynucleotides requires TLR9 either in the host or in the tumor itself. J Immuno/ 2007, 179(4): 2493-2500.

17. Jahrsdorfer B, Weiner GJ: CpG oligodeoxynucleotides as immunotherapy in cancer. Update Cancer Ther 2008, 3(1):27-32.

18. Weiner GJ: CpG oligodeoxynucleotide-based therapy of lymphoid malignancies. Adv Drug Deliv Rev 2009, 61(3):263-267.

19. Galea I, Bechmann I, Perry VH: What is immune privilege (not)? Trends Immunol 2007, 28(1):12-18.

\section{doi:10.1186/1756-9966-32-18}

Cite this article as: Ben Abdelwahed et al:: Lymphoma B-cell responsiveness to $\mathrm{CpG}$-DNA depends on the tumor microenvironment. Journal of Experimental \& Clinical Cancer Research 2013 32:18. 